\title{
Measuring the Absolute Photo Detection Efficiency Using Photon Number Correlations
}

\author{
Michael Lindenthal and Johannes Kofler \\ Institut für Experimentalphysik, Universität Wien, 1090 Wien, Austria \\ Institut für Quantenoptik und Quanteninformation, \\ Österreichische Akademie der Wissenschaften, 1090 Wien, Austria
}

(Dated: July 25, 2018)

\begin{abstract}
We present two methods for determining the absolute detection efficiency of photon-counting detectors directly from their singles rates under illumination from a nonclassical light source. One method is based on a continuous variable analogue to coincidence counting in discrete photon experiments, but does not actually rely on high detector time resolutions. The second method is based on difference detection which is a typical detection scheme in continuous variable quantum optics experiments. Since no coincidence detection is required with either method, they are useful for detection efficiency measurements of photo detectors with detector time resolutions far too low to resolve coincidence events.
\end{abstract}

\section{INTRODUCTION}

High detection efficiency is crucial in many contexts within quantum optics. In particular, recent linear optics quantum computation methods have been shown to require detection efficiencies higher than $90 \%$ to be scalable [1, 2, 3, 4]. Another well known example comes from Bell inequality experiments where a detection efficiency of at least $2 / 3$ is required to close the detection loophole 5. Thus higher detector efficiencies do not only have the obvious advantage of providing more signal, but are also of great relevance for both current quantum information science and fundamental quantum physics. Therefore building new high efficiency detectors is a crucial area of research. In this article we present methods to evaluate their absolute detection efficiency. The typical way to measure detection efficiency is to use a calibrated reference detector, and to compare its response to an incident light beam of constant intensity with the response of the detector under test. It has been shown that quantum mechanics itself provides a way to measure the absolute detection efficiency of light detectors without the need for a reference detector. By using non-classical photon statistics such as those produced in spontaneous parametric down conversion [6] both theory and experiment show that the absolute detection efficiency can be determined by the ratio of the coincidence rate to the singles rate [7, 8, 9]. However, this method requires the detectors to have a high enough time resolution for coincidence counting. We provide work-around schemes that overcome this limitation. Specifically, we have developed two related detector efficiency calibration methods that use quantum correlated light but do not rely on high time resolution coincidence counting [10]. This might be of particular interest for novel detectors and prototypes which are not yet capable of coincidence counting, such as electron multiplying CCD cameras.

The first method is based on a continuous variable analogue of coincidence counting in discrete photon experiments. Recall that coincidence counting is essentially bitwise multiplication (i.e., an AND gate) of single counts within a small coincidence window. In the continuous variable limit, this is achieved by measuring the mean product of the detected photon numbers in two beams generated by spontaneous parametric down conversion. Since down conversion photons are emitted in pairs, the normalized mean product of the photon numbers measured in the two beams during a specific time is maximal in the case of perfect detection efficiencies when all photons are detected. On the other hand, in the limit of small detection efficiencies, the normalized mean product of the detected photon numbers has its minimum. For the general case an expression can be derived, that allows calculation of detection efficiencies from the measured mean product of the detected photon numbers in the two down conversion beams.

The second method uses the variance of the detected photon number differences in two beams generated by spontaneous parametric down conversion. Such difference detection is a typical detection scheme in continuous variable quantum optics experiments 11, 12. Since the photons in the two beams are perfectly correlated, the difference in the photon numbers measured in the two beams is zero in the case of perfect detection efficiencies when all photons are detected. Uncorrelated loss in these two beams diminishes those perfect correlations. Therefore in the limit of small detection efficiencies, the normalized variance of the differences in the detected photon numbers has its maximum. For the general case again an expression can be derived, that allows to calculate the detection efficiencies from the measured variance of the detected photon number differences in the two down conversion beams.

The article is structured as follows: First we derive these relations mentioned above disregarding background. Then we generalize these results to include two different background levels in the two down conversion beams, since background light is a very significant contribution in single photon counting experiments. 


\section{THEORY}

\section{A. Product Detection Method}

We begin by deriving a relationship between the mean product of the singles rates and the detection efficiencies in each down conversion beam, $\eta_{1}$ and $\eta_{2}$, in the absence of background. We assume that parametric down conversion emits light beams described by a general distribution $G_{N}(k)$ of the number of photon pairs $k$ with mean value $\langle k\rangle_{G}=N$ and the second moment $\left\langle k^{2}\right\rangle_{G}$ [13]. The probability of detecting $l$ out of $k$ photons in each of the two down conversion beams is given by the binomial distribution $B_{k, \eta_{i}}(l)=\left(\begin{array}{l}k \\ l\end{array}\right) \eta_{i}^{l}\left(1-\eta_{i}\right)^{k-l}$, with mean value $\langle l\rangle_{B}=\eta_{i} k$ and the second moment $\left\langle l^{2}\right\rangle_{B}=\eta_{i} k-\eta_{i}^{2} k+\eta_{i}^{2} k^{2}(i=1,2)$. Thus the mean product of the detected photon numbers in the two down conversion beams is given by

$$
\begin{aligned}
\langle l m\rangle & =\sum_{k=0}^{\infty} \sum_{l=0}^{k} \sum_{m=0}^{k} G_{N}(k) B_{k, \eta_{1}}(l) B_{k, \eta_{2}}(m) l m \\
& =\eta_{1} \eta_{2}\left\langle k^{2}\right\rangle_{G}=\frac{\eta_{2}}{\eta_{1}}\left(\left\langle l^{2}\right\rangle-\langle l\rangle+\eta_{1}\langle l\rangle\right),
\end{aligned}
$$

where $l$ and $m$ are the detected photon numbers in each down conversion beam, $\langle l\rangle=\eta_{1}\langle k\rangle_{G}$ and $\left\langle l^{2}\right\rangle=\eta_{1}\langle k\rangle_{G}-$ $\eta_{1}^{2}\langle k\rangle_{G}+\eta_{1}^{2}\left\langle k^{2}\right\rangle_{G}$.

Together with the expression $\frac{\langle l\rangle}{\langle m\rangle}=\frac{\eta_{1}}{\eta_{2}}$, the detection efficiency $\eta_{1}$ follows as

$$
\eta_{1}=\frac{\langle l m\rangle}{\langle m\rangle}-\frac{\left\langle l^{2}\right\rangle}{\langle l\rangle}+1
$$

The corresponding result for $\eta_{2}$ is

$$
\eta_{2}=\frac{\langle l m\rangle}{\langle l\rangle}-\frac{\left\langle m^{2}\right\rangle}{\langle m\rangle}+1
$$

Note that neither formula depends on the coincidence rate. However, the quantum statistics of the light enters the expressions in the mean product of the singles rates.

In a former method [7] the absolute detection efficiency is determined from the ratio of the mean coincidence rate $\langle c\rangle$ to the mean singles rate $\langle l\rangle$ or $\langle m\rangle, \eta_{1}=\frac{\langle c\rangle}{\langle m\rangle}$ and $\eta_{2}=\frac{\langle c\rangle}{\langle l\rangle}$. Together with (2) or (3) one obtains for $\eta=\eta_{1}=\eta_{2}$, and hence $\langle s\rangle=\langle l\rangle=\langle m\rangle$ and $\left\langle s^{2}\right\rangle=\left\langle l^{2}\right\rangle=\left\langle m^{2}\right\rangle$,

$$
\langle s\rangle-\langle c\rangle=\left\langle s^{2}\right\rangle-\langle l m\rangle=\frac{\left\langle(l-m)^{2}\right\rangle}{2} .
$$

This simple expression relates the difference between the mean singles rate and the mean coincidence rate to the variance of the detected photon number differences in the two down conversion beams. This motivates our second approach for determining the detection efficiencies from the variance of the detected photon number differences in the two down conversion beams described in section IC

\section{B. Product Detection Method: General Approach Including Background}

We now extend this theory to cover more realistic experimental conditions and correct the measurements for possibly different backgrounds in the two detectors. The averaged quantities contained in (2) and (3) have to be extracted from experimentally accessible quantities which include background. We do so by splitting up the measured photon numbers (subscript $\mathrm{M}$ ) into the photon numbers corresponding to the signal (no subscript) and into photon numbers corresponding to the background (subscript B), where $l=l_{M}-l_{B}$ and $m=m_{M}-m_{B}$. The background can be estimated experimentally from a separate configuration. We get

$$
\begin{aligned}
\langle l\rangle & =\left\langle l_{M}\right\rangle-\left\langle l_{B}\right\rangle, \\
\langle m\rangle & =\left\langle m_{M}\right\rangle-\left\langle m_{B}\right\rangle, \\
\left\langle l^{2}\right\rangle & =\left\langle l_{M}^{2}\right\rangle-\left\langle l_{B}^{2}\right\rangle-2\left\langle l_{M}\right\rangle\left\langle l_{B}\right\rangle+2\left\langle l_{B}\right\rangle^{2} \\
\left\langle m^{2}\right\rangle & =\left\langle m_{M}^{2}\right\rangle-\left\langle m_{B}^{2}\right\rangle-2\left\langle m_{M}\right\rangle\left\langle m_{B}\right\rangle+2\left\langle m_{B}\right\rangle^{2}
\end{aligned}
$$


and

$$
\langle l m\rangle=\left\langle l_{M} m_{M}\right\rangle-\left\langle l_{M}\right\rangle\left\langle m_{B}\right\rangle-\left\langle l_{B}\right\rangle\left\langle m_{M}\right\rangle+\left\langle l_{B}\right\rangle\left\langle m_{B}\right\rangle .
$$

Here we used the statistical independence of $l$ and $l_{B}$ and $m$ and $m_{B}$, respectively. By inserting these expressions into (2) and (3) the detection efficiencies can be determined from the data directly measurable in an experiment:

$$
\eta_{1}=\frac{\left\langle l_{M} m_{M}\right\rangle-\left\langle l_{M}\right\rangle\left\langle m_{B}\right\rangle-\left\langle l_{B}\right\rangle\left\langle m_{M}\right\rangle+\left\langle l_{B}\right\rangle\left\langle m_{B}\right\rangle}{\left\langle m_{M}\right\rangle-\left\langle m_{B}\right\rangle}-\frac{\left\langle l_{M}^{2}\right\rangle-\left\langle l_{B}^{2}\right\rangle-2\left\langle l_{M}\right\rangle\left\langle l_{B}\right\rangle+2\left\langle l_{B}\right\rangle^{2}}{\left\langle l_{M}\right\rangle-\left\langle l_{B}\right\rangle}+1
$$

and

$$
\eta_{2}=\frac{\left\langle l_{M} m_{M}\right\rangle-\left\langle l_{M}\right\rangle\left\langle m_{B}\right\rangle-\left\langle l_{B}\right\rangle\left\langle m_{M}\right\rangle+\left\langle l_{B}\right\rangle\left\langle m_{B}\right\rangle}{\left\langle l_{M}\right\rangle-\left\langle l_{B}\right\rangle}-\frac{\left\langle m_{M}^{2}\right\rangle-\left\langle m_{B}^{2}\right\rangle-2\left\langle m_{M}\right\rangle\left\langle m_{B}\right\rangle+2\left\langle m_{B}\right\rangle^{2}}{\left\langle m_{M}\right\rangle-\left\langle m_{B}\right\rangle}+1 .
$$

\section{Difference Detection Method}

Our second approach for determining the absolute detection efficiencies in each down conversion beam, $\eta_{1}$ and $\eta_{2}$, relies on measuring the variance of the differences in the singles rates $\left\langle(l-m)^{2}\right\rangle$. This method is closely related to the approach described in section IA since $\left\langle(l-m)^{2}\right\rangle=\left\langle l^{2}\right\rangle+\left\langle m^{2}\right\rangle-2\langle l m\rangle$, and both $\left\langle(l-m)^{2}\right\rangle$ and $\langle l m\rangle$ depend on the degree of second-order coherence which is affected by uncorrelated loss. However, the two methods may be useful under different circumstances, especially since difference detection is a typical detection scheme in continuous variable quantum optics experiments.

The variance of the detected photon number differences in the two down conversion beams is given by

$$
\begin{aligned}
\left\langle(l-m)^{2}\right\rangle & =\sum_{k=0}^{\infty} \sum_{l=0}^{k} \sum_{m=0}^{k} G_{N}(k) B_{k, \eta_{1}}(l) B_{k, \eta_{2}}(m)(l-m)^{2} \\
& =\langle l\rangle+\langle m\rangle-\left(\eta_{1}\langle l\rangle+\eta_{2}\langle m\rangle\right)+\left(\eta_{1}-\eta_{2}\right)^{2} \frac{\left\langle l^{2}\right\rangle-\langle l\rangle+\eta_{1}\langle l\rangle}{\eta_{1}^{2}},
\end{aligned}
$$

where $l$ and $m$ are the detected photon numbers in each down conversion beam.

Together with the expression $\frac{\langle l\rangle}{\langle m\rangle}=\frac{\eta_{1}}{\eta_{2}}$, the detection efficiency $\eta_{1}$ follows as

$$
\eta_{1}=\frac{3\langle m\rangle-\frac{\langle m\rangle^{2}}{\langle l\rangle}+\left\langle l^{2}\right\rangle\left(1-\frac{\langle m\rangle}{\langle l\rangle}\right)^{2}-\left\langle(l-m)^{2}\right\rangle}{2\langle m\rangle} .
$$

Correspondingly,

$$
\eta_{2}=\frac{3\langle l\rangle-\frac{\langle l\rangle^{2}}{\langle m\rangle}+\left\langle m^{2}\right\rangle\left(1-\frac{\langle l\rangle}{\langle m\rangle}\right)^{2}-\left\langle(l-m)^{2}\right\rangle}{2\langle l\rangle} .
$$

For $\eta=\eta_{1}=\eta_{2}$, and hence $\langle s\rangle=\langle l\rangle=\langle m\rangle$, (12) becomes

$$
\left\langle(l-m)^{2}\right\rangle=2(1-\eta)\langle s\rangle .
$$

For perfect detection efficiencies $(\eta=1),\left\langle(l-m)^{2}\right\rangle=0$. Since the down-converted photons are always created in pairs, the difference in the detected photon number, and hence the variance of that quantity, is exactly zero if all of the pairs are detected. In the limit of very small detection efficiencies $(\eta \ll 1),\left\langle(l-m)^{2}\right\rangle \approx 2\langle s\rangle$, which corresponds to the variance of two independent Poissonian light beams of equal intensities. The non-Poissonian contributions in $G_{N}(k)$ cancel out, showing that quantum statistics strongly depends on the detection efficiency of the detectors.

Expression (15) can be rewritten as

$$
\eta=1-\frac{\left\langle(l-m)^{2}\right\rangle}{2\langle s\rangle} .
$$

A similar expression was stated without explicit derivation previously 11, 12.

With $\langle s\rangle=\eta N$, the normalized expression (15) reads $\frac{\left\langle(l-m)^{2}\right\rangle}{\langle s\rangle^{2}}=\frac{2}{N}\left(\frac{1}{\eta}-1\right)$, which verifies that the normalized variance of the detected photon number differences in the two down conversion beams diverges for $\eta \rightarrow 0$ and goes to zero for $\eta \rightarrow 1$. Using equation (4), an analogous treatment can be performed for the normalized mean product of the detected photon numbers in the two beams. 


\section{Difference Detection Method: General Approach Including Background}

As in section $\llbracket \mathrm{B}$ we extend this theory to cover the more realistic experimental conditions including background. The quantities in (13) and (14) have to be extracted from quantities that are directly accessible to measurement. We get (5) - (8) and

$$
\left\langle(l-m)^{2}\right\rangle=\left\langle\left(l_{M}-m_{M}\right)^{2}\right\rangle+2\left(\left\langle l_{B}\right\rangle-\left\langle m_{B}\right\rangle\right)^{2}-2\left(\left\langle l_{M}\right\rangle-\left\langle m_{M}\right\rangle\right)\left(\left\langle l_{B}\right\rangle-\left\langle m_{B}\right\rangle\right)+2\left\langle l_{B}\right\rangle\left\langle m_{B}\right\rangle-\left\langle l_{B}^{2}\right\rangle-\left\langle m_{B}^{2}\right\rangle
$$

for the background corrected difference term.

By inserting (5) -(8) and (17) into (13) and (14) the detection efficiencies can be determined from the data directly measurable in an experiment,

$$
\begin{aligned}
\eta_{1}= & \frac{3\left(\left\langle m_{M}\right\rangle-\left\langle m_{B}\right\rangle\right)-\frac{\left(\left\langle m_{M}\right\rangle-\left\langle m_{B}\right\rangle\right)^{2}}{\left\langle l_{M}\right\rangle-\left\langle l_{B}\right\rangle}}{2\left(\left\langle m_{M}\right\rangle-\left\langle m_{B}\right\rangle\right)}+\frac{\left\langle l_{M}^{2}\right\rangle-\left\langle l_{B}^{2}\right\rangle-2\left\langle l_{M}\right\rangle\left\langle l_{B}\right\rangle+2\left\langle l_{B}\right\rangle^{2}}{2\left(\left\langle m_{M}\right\rangle-\left\langle m_{B}\right\rangle\right)}\left(1-\frac{\left\langle m_{M}\right\rangle-\left\langle m_{B}\right\rangle}{\left\langle l_{M}\right\rangle-\left\langle l_{B}\right\rangle}\right)^{2} \\
& -\frac{\left\langle\left(l_{M}-m_{M}\right)^{2}\right\rangle+2\left(\left\langle l_{B}\right\rangle-\left\langle m_{B}\right\rangle\right)^{2}}{2\left(\left\langle m_{M}\right\rangle-\left\langle m_{B}\right\rangle\right)}-\frac{2\left(\left\langle l_{M}\right\rangle-\left\langle m_{M}\right\rangle\right)\left(\left\langle l_{B}\right\rangle-\left\langle m_{B}\right\rangle\right)}{2\left(\left\langle m_{M}\right\rangle-\left\langle m_{B}\right\rangle\right)}+\frac{2\left\langle l_{B}\right\rangle\left\langle m_{B}\right\rangle-\left\langle l_{B}^{2}\right\rangle-\left\langle m_{B}^{2}\right\rangle}{2\left(\left\langle m_{M}\right\rangle-\left\langle m_{B}\right\rangle\right)} .
\end{aligned}
$$

and

$$
\begin{aligned}
\eta_{2}= & \frac{3\left(\left\langle l_{M}\right\rangle-\left\langle l_{B}\right\rangle\right)-\frac{\left(\left\langle l_{M}\right\rangle-\left\langle l_{B}\right\rangle\right)^{2}}{\left\langle m_{M}\right\rangle-\left\langle m_{B}\right\rangle}}{2\left(\left\langle l_{M}\right\rangle-\left\langle l_{B}\right\rangle\right)}+\frac{\left\langle m_{M}^{2}\right\rangle-\left\langle m_{B}^{2}\right\rangle-2\left\langle m_{M}\right\rangle\left\langle m_{B}\right\rangle+2\left\langle m_{B}\right\rangle^{2}}{2\left(\left\langle l_{M}\right\rangle-\left\langle l_{B}\right\rangle\right)}\left(1-\frac{\left\langle l_{M}\right\rangle-\left\langle l_{B}\right\rangle}{\left\langle m_{M}\right\rangle-\left\langle m_{B}\right\rangle}\right)^{2} \\
& -\frac{\left\langle\left(l_{M}-m_{M}\right)^{2}\right\rangle+2\left(\left\langle l_{B}\right\rangle-\left\langle m_{B}\right\rangle\right)^{2}}{2\left(\left\langle l_{M}\right\rangle-\left\langle l_{B}\right\rangle\right)}-\frac{2\left(\left\langle l_{M}\right\rangle-\left\langle m_{M}\right\rangle\right)\left(\left\langle l_{B}\right\rangle-\left\langle m_{B}\right\rangle\right)}{2\left(\left\langle l_{M}\right\rangle-\left\langle l_{B}\right\rangle\right)}+\frac{2\left\langle l_{B}\right\rangle\left\langle m_{B}\right\rangle-\left\langle l_{B}^{2}\right\rangle-\left\langle m_{B}^{2}\right\rangle}{2\left(\left\langle l_{M}\right\rangle-\left\langle l_{B}\right\rangle\right)} .
\end{aligned}
$$

\section{E. Error Estimates}

Finally, we want to derive the statistical errors of the detection efficiencies (without background), i.e., $(A)$ for the product detection, eq. (2), $(B)$ for the difference detection, eq. (13), and $(C)$ for the coincidence method [7], described above eq. (4):

$$
\begin{aligned}
& \eta_{1}^{(A)}=\frac{\langle l m\rangle}{\langle m\rangle}-\frac{\left\langle l^{2}\right\rangle}{\langle l\rangle}+1 \\
& \eta_{1}^{(B)}=\frac{3\langle m\rangle-\frac{\langle m\rangle^{2}}{\langle l\rangle}+\left\langle l^{2}\right\rangle\left(1-\frac{\langle m\rangle}{\langle l\rangle}\right)^{2}-\left\langle(l-m)^{2}\right\rangle}{2\langle m\rangle}, \\
& \eta_{1}^{(C)}=\frac{\langle c\rangle}{\langle m\rangle},
\end{aligned}
$$

In each case $\eta_{1}$ is a function of several mean values, i.e., $\eta_{1}=\eta_{1}(\langle u\rangle,\langle v\rangle, \ldots)$ where $u, v, \ldots \in\left\{l, m, l^{2}, l m,(l-m)^{2}, c\right\}$. The sample variance of $\eta_{1}$ is defined as

$$
\sigma^{2}\left(\eta_{1}\right) \equiv \sigma_{\langle u\rangle}^{2}\left(\frac{\partial \eta_{1}}{\partial\langle u\rangle}\right)^{2}+\sigma_{\langle v\rangle}^{2}\left(\frac{\partial \eta_{1}}{\partial\langle v\rangle}\right)^{2}+2 \sigma_{\langle u\rangle\langle v\rangle} \frac{\partial \eta_{1}}{\partial\langle u\rangle} \frac{\partial \eta_{1}}{\partial\langle v\rangle}+\ldots
$$

where

$$
\begin{aligned}
\sigma_{\langle u\rangle}^{2} & \equiv \frac{\left\langle u^{2}\right\rangle-\langle u\rangle^{2}}{M}, \\
\sigma_{\langle u\rangle\langle v\rangle} & \equiv \frac{\langle u v\rangle-\langle u\rangle\langle v\rangle}{M}
\end{aligned}
$$

are the variances and covariances of the sample means with sample size $M$. It has to be stressed that in experiments the time interval chosen for accumulating the individual measurements needs to be much larger than the resolving time of the detector under test. The mean values are given by

$$
\langle x\rangle=\sum_{k=0}^{\infty} \sum_{l=0}^{k} \sum_{m=0}^{k} G_{N}(k) B_{k, \eta_{1}}(l) B_{k, \eta_{2}}(m) x .
$$



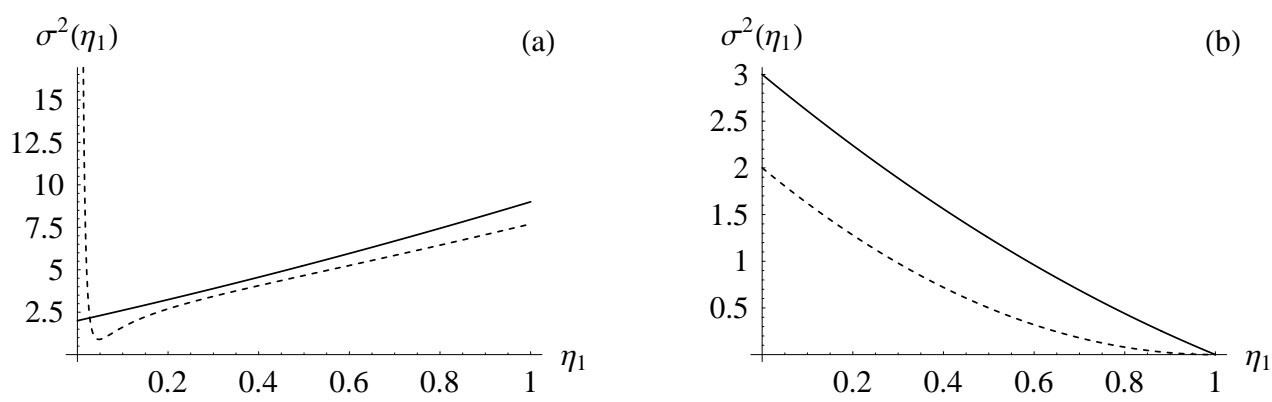

FIG. 1: Sample variances $\sigma^{2}\left(\eta_{1}^{(A)}\right)$ (product method, solid line) and $\sigma^{2}\left(\eta_{1}^{(B)}\right)$ (difference method, dashed line) as a function of $\eta_{1}$ for a Poissonian distribution in the limit $N \gg 1$. For the sake of generality the sample size is $M=1$, where all variances scale with $1 / M$. (a) The second efficiency is constant: $\eta_{2}=0.1$. The variance $\sigma^{2}\left(\eta_{1}^{(B)}\right)$ diverges for $\eta_{1} \rightarrow 0$. (b) Equal efficiencies: $\eta_{2}=\eta_{1}$.

Due to the perfect correlations the first and second moment of the coincidences can be computed by applying the binomial distribution to one down conversion arm twice:

$$
\left\langle c^{p}\right\rangle=\sum_{k=0}^{\infty} \sum_{l=0}^{k} \sum_{m=0}^{l} G_{N}(k) B_{k, \eta_{1}}(l) B_{k, \eta_{2}}(m) m^{p},
$$

where $p=1,2$.

For a Poissonian down conversion distribution $G_{N}(k)=N^{k} \exp (-N) / k$ ! with $N$ the expected mean photon number in one sample measurement, the efficiency sample variances in the three cases are

$$
\begin{aligned}
\sigma^{2}\left(\eta_{1}^{(A)}\right) & =\frac{\eta_{1}\left(1+N-\eta_{1}\right)+N \eta_{2}\left[2+\eta_{1}\left(\eta_{1}-4\right)\right]}{M N \eta_{2}} \\
\sigma^{2}\left(\eta_{1}^{(B)}\right) & =\frac{2 \eta_{1}^{4}\left(N \eta_{2}-1\right)+2 \eta_{1}^{3}\left[1+N\left(1+2 \eta_{2}\left(\eta_{2}-3\right)\right)\right]+N \eta_{1}^{2} \eta_{2}\left[5-2 \eta_{2}\left(\eta_{2}-2\right)\right]-4 N \eta_{1} \eta_{2}^{2}+N \eta_{2}^{3}}{2 M N \eta_{1}^{2} \eta_{2}} \\
\sigma^{2}\left(\eta_{1}^{(C)}\right) & =\frac{\eta_{1}\left(1+\eta_{1}-2 \eta_{1} \eta_{2}\right)}{M N \eta_{2}}
\end{aligned}
$$

In the limit $N \gg 1$ the variance for the coincidence method vanishes: $\sigma^{2}\left(\eta_{1}^{(C)}\right) \rightarrow 0$. For the product and difference detection the variances approach constant values which depend on the efficiencies $\eta_{1}$ and $\eta_{2}$ as well as the sample size $M$. Figure 1 shows these two variances as a function of $\eta_{1}$ for $M=1$ where all sample variances scale inversely with the sample size. In general, the difference method is more accurate than the product method, except in the case of fixed $\eta_{2}$ and vanishing $\eta_{1}$. In the special case of equal efficiencies $\eta_{1}=\eta_{2}$ the expression for the difference method simplifies tremendously:

$$
\left.\sigma^{2}\left(\eta_{1}^{(B)}\right)\right|_{\eta_{2}=\eta_{1}}=\frac{2\left(1-\eta_{1}\right)^{2}}{M}
$$

For a thermal distribution $G_{N}(k) \propto \exp (-k / N)$ the first two variances are more cumbersome and we do not write them here. In the limit of increasing $N$ the variance for the coincidence method $\sigma^{2}\left(\eta_{1}^{(C)}\right)$ vanishes again, while the variances for the product and difference detection method linearly diverge in the limit $N \gg 1$, still also scaling with $1 / M$. Only in the special case of equal efficiencies $\eta_{1}=\eta_{2}$ the variance for the difference method $\sigma^{2}\left(\eta_{1}^{(B)}\right)$ becomes independent of $N$. Therefore, if equal detectors are used, the difference method is more favorable. In this special case and for $N \gg 1$ we have the simple expression

$$
\left.\sigma^{2}\left(\eta_{1}^{(B)}\right)\right|_{\eta_{2}=\eta_{1}}=\frac{4\left(1-\eta_{1}\right)^{2}}{M}
$$

Hence, in this case, the variance for the thermal distribution has the same form as expression (31) for the Poissonian distribution. 


\section{CONCLUSIONS AND OUTLOOK}

We have presented two methods for determining the absolute detection efficiency of photo detectors. Since they are applicable to detectors with low time resolution, they overcome the limitations typical for absolute detection efficiency measurements. The first is based on measuring the mean product of the detected singles rates in two beams generated by spontaneous parametric down conversion. The second method uses the variance measurements of the differences in the detected singles rates in the two down conversion beams. The two methods correspond to the different detection methods typically used in either the discrete photon or continuous variable communities, respectively. Both procedures could be used for measuring the absolute detection efficiency of photo detectors that do not provide the appropriate time resolution for coincidence counting.

\section{Acknowledgments}

This work was funded by the DOC-program of the Austrian Academy of Sciences as well as the Austrian Science Foundation (FWF), Project SFB 1506. We thank Kevin Resch, Markus Aspelmeyer, Aephraim Steinberg, Andrew White and Gregor Weihs for helpful and motivating discussions. Especially we thank Anton Zeilinger for supporting this work.

[1] S. Gasparoni, J.-W. Pan, P. Walther, T. Rudolph, and A. Zeilinger, "Realization of a photonic controlled-not gate sufficient for quantum computation," Phys. Rev. Lett. 93, 020504 (2004).

[2] E. Knill, R. Laflamme, and G. J. Milburn, "A scheme for effcient quantum computation with linear optics," Nature 409, $46(2001)$.

[3] J. L. O'Brien, G. J. Pryde, A. G. White, T. C. Ralph, and D. Branning, "Demonstration of an all-optical quantum controlled-not gate," Nature 426, 264 (2003).

[4] K. Sanaka, T. Jennewein, J.-W. Pan, K. Resch, and A. Zeilinger, "Experimental nonlinear sign shift for linear optics quantum computation," Phys. Rev. Lett. 92, 017902 (2004).

[5] P. H. Eberhard, "Background level and counter efficiencies required for a loophole-free einstein-podolsky-rosen experiment," Phys. Rev. A 47, R747 (1993).

[6] P. G. Kwiat, K. Mattle, H. Weinfurter, A. Zeilinger, A. V. Sergienko, and Y. Shih, "New high-intensity source of polarization-entangled photon pairs," Phys. Rev. Lett. 75, 4337 (1995).

[7] D. N. Klyshko, "Use of two-photon light for absolute calibration of photoelectric detectors," Sov. J. Quantum Electron. 10, $1112(1980)$.

[8] P. G. Kwiat, A. M. Steinberg, R. Y. Chiao, P. H. Eberhard, and M. D. Petroff, "Absolute efficiency and time-response measurement of single-photon detectors," Appl. Opt. 33, 1844 (1994).

[9] J. G. Rarity, K. D. Ridley, and P. R. Tapster, "Absolute measurement of detector quantum efficiency using parametric downconversion," Appl. Opt. 26, 4616 (1987).

[10] Similar investigations were independently and simultaneously performed in G. Brida, M. Chekhova, M. Genovese, A. Penin, and I. Ruo-Berchera, "The possibility of absolute calibration of analog detectors by using parametric down-conversion: a systematical study," arXiv:quant-ph/0511093 (2005).

[11] O. Aytür and P. Kumar, "Pulsed twin beams of light," Phys. Rev. Lett. 65, 1551 (1990).

[12] A. Heidmann, R. J. Horowicz, S. Reynaud, E. Giacobino, C. Fabre, and G. Camy, "Observation of quantum noise reduction on twin laser beams," Phys. Rev. Lett. 59, 2555 (1987).

[13] Sources of spontaneous parametric down conversion are known to follow a thermal distribution. However, this does not play a crucial role in the present derivation of the efficiencies themselves but is only important for the error estimates (see section (IE). For thermal photon statistics in spontaneous parametric down conversion see L. Mandel and E. Wolf, Optical Coherence and Quantum Optics, Chap. 14 (Cambridge University Press, 1995). 